\title{
SPECTRUM OF THE JACOBI TAU APPROXIMATION FOR THE SECOND DERIVATIVE OPERATOR
}

\author{
MARIOS CHARALAMBIDES* AND FABIAN WALEFFE ${ }^{\dagger}$
}

\begin{abstract}
It is proved that the eigenvalues of the Jacobi Tau method for the second derivative operator with Dirichlet boundary conditions are real, negative and distinct for a range of the Jacobi parameters. Special emphasis is placed on the symmetric case of the Gegenbauer Tau method where the range of parameters included in the theorems can be extended and characteristic polynomials given by successive order approximations interlace. This includes the common Chebyshev and Legendre, Tau and Galerkin methods. The characteristic polynomials for the Gegenbauer Tau method are shown to obey three term recurrences plus a constant term which vanishes for the Legendre Tau and Galerkin cases. These recurrences are equivalent to a tridiagonal plus one row matrix structure. The spectral integration formulation of the Gegenbauer Tau method is shown to lead directly to that fundamental and well-conditioned tridiagonal plus one row matrix structure. A Matlab code is provided.
\end{abstract}

Key words. Jacobi polynomials, Gegenbauer polynomials, stable polynomials, positive pairs, zeros of polynomials, spectral methods

AMS subject classifications. 65D30, 65L10, 65L15, 65M70, 65N35, 26C10

1. Introduction. Constructing polynomial approximations to solutions of differential equations is the basic ingredient of most numerical methods. Approximations based on orthogonal polynomials have been widely used (e.g. [2], [3], [8]) because their rate of convergence is faster than algebraic for arbitrary boundary conditions when the solution is smooth. The purpose of this paper is to give a rigorous proof that the spectrum of the Jacobi Tau approximation is real, negative and distinct for the second order operator with Dirichlet boundary conditions. The Jacobi Tau class of spectral methods includes the common Chebyshev and Legendre Tau and Galerkin formulations, as demonstrated below. The general method of proof is similar to that used by Gottlieb and Lustman [6, 7] to prove such results for the Chebyshev collocation operator. However, we argue in section 3.1 that Gottlieb and Lustman's proof for the collocation operator is not complete.

The spectrum of Jacobi Tau approximation for the 1st order operator has been considered elsewhere 4. Here, we consider polynomial approximations to the eigenvalue problem

$$
\frac{d^{2} u}{d x^{2}}=\lambda u \quad-1<x<1 \quad \text { with } \quad u( \pm 1)=0 .
$$

The spectrum of Jacobi polynomial approximations to this eigenvalue problem is directly relevant to numerical simulations of the diffusion equation $u_{t}=u_{x x}$ which is itself a building block for numerical solution of various other problems including the Stokes and Navier-Stokes equations (e.g. [3, §5.1], [18).

The 2nd order problem (1.1) is a self-adjoint, negative definite Sturm-Liouville differential eigenproblem, so its eigenvalues $\lambda$ are real, negative and distinct. The

\footnotetext{
${ }^{*}$ Department of Business Administration, Frederick Institute of Technology, 7 Yianni Frederickou Street, Pallouriotissa, PO Box 24729, 1303 Nicosia, Cyprus (bus.chm@fit.ac.cy).

$\dagger$ Department of Mathematics, University of Wisconsin, Madison, WI 53706, USA (waleffe@math.wisc.edu). This work was supported in part by NSF grant DMS-0204636. [Preprint Jul 24, 2006]
} 
eigenmodes separate into even and odd modes and have the simple exact expressions

$$
\begin{array}{ll}
u_{e}(x)=\cos (2 k-1) \frac{\pi}{2} x, & \lambda=-(2 k-1)^{2} \frac{\pi^{2}}{4}, \\
u_{o}(x)=\sin k \pi x, & \lambda=-k^{2} \pi^{2} .
\end{array}
$$

for $k=1,2,3, \ldots$.

If $u_{n}(x)$ is a polynomial approximation of degree $n$ to the exact solution $u(x)$, then $u_{n}(x)$ satisfies the following differential equation

$$
\lambda u_{n}(x)-D^{2} u_{n}(x)=R_{n}(x)
$$

where the residual $R_{n}(x)$ is a polynomial of degree $n$ in $x$ and $D=d / d x$. We can invert this relation to express the polynomial approximation $u_{n}(x)$ in terms of the residual $R_{n}(x)$ []

$$
u_{n}(x)=\mu \sum_{k=0}^{[n / 2]} \mu^{k} D^{2 k} R_{n}(x)
$$

where $\mu=1 / \lambda$, and $[n / 2]$ denotes the greatest integer less or equal to $n / 2$. We can assume that $\lambda \neq 0$ because $\lambda=0$ with $u_{n}( \pm 1)=0$ necessarily corresponds to the trivial solution $u_{n}(x)=0, \forall x$ in $[-1,1]$, as shown below. The inversion (1.4) follows from formal application of the geometric (Neumann) series for $\left(1-\mu D^{2}\right)^{-1}=$ $\sum_{k=0}^{\infty} \mu^{k} D^{2 k}$ which terminates since $R_{n}(x)$ is a polynomial. That inversion can also be derived by repeated application of the operator $\mu D^{2}$ to equation (1.3). Summation of the resulting suite of equations leads to (1.4) thanks to telescopic cancelations on the left hand side.

Spectral methods fit in the general framework of the method of weighted residuals 3]. In the Tau method [3, $\$ 10.4 .2]$, the polynomial approximation $u_{n}(x)$ is determined from the boundary conditions $u_{n}( \pm 1)=0$ and the requirement that $R_{n}(x)$ is orthogonal to all polynomials $p_{n-2}(x)$ of degree $n-2$ or less with respect to a weight function $W(x) \geq 0$ in the interval $(-1,1)$

$$
\int_{-1}^{1} R_{n}(x) p_{n-2}(x) W(x) d x=0 .
$$

These requirements provide $n+1$ equations for the $n+1$ undetermined constants in the polynomial approximation $u_{n}(x)$. For the Jacobi weight function $W_{\alpha, \beta}(x)=$ $(1-x)^{\alpha}(1+x)^{\beta}$, the residual (1.3) can be written as

$$
R_{n}(x)=\tau_{0} \lambda P_{n}^{(\alpha, \beta)}(x)+\tau_{1} \lambda P_{n-1}^{(\alpha, \beta)}(x)
$$

for some $x$-independent coefficients $\tau_{0}$ and $\tau_{1}$, where $P_{n}^{(\alpha, \beta)}(x)$ is the Jacobi polynomial of degree $n$ (sect. B.11). This follows from orthogonality of the Jacobi polynomials in $-1<x<1$ with respect to the Jacobi weight $W_{\alpha, \beta}(x)$ which implies orthogonality of the Jacobi polynomial of degree $k$ to any polynomial of degree $k-1$ or less with respect to that weight function. Jacobi polynomials are the most general class of polynomial solutions of a Sturm-Liouville eigenproblem that is singular at \pm 1 as required for faster than algebraic convergence [3, $\S 9.2 .2, \S 9.6 .1]$. It is now easy to verify from (1.3) and (1.6) that if $\lambda=0$ then $D^{2} u_{n}(x)=R_{n}(x)=0$ for all $x$ in $(-1,1)$ but 
the boundary conditions $u_{n}( \pm 1)=0$ would then require that $u_{n}(x)=0$ for all $x$ in $[-1,1]$. Therefore we can assume that $\lambda \neq 0$.

In the Galerkin approach, $u_{n}(x)$ is determined from the boundary conditions $u_{n}( \pm 1)=0$ and orthogonality of the residual $R_{n}(x)$ to all polynomials of degree $n$ that vanish at $x= \pm 1$, with respect to a weight function $W(x) \geq 0$. In other words, the test functions are in the same space (polynomials of degree $n$ ) as the trial functions and they satisfy the same boundary conditions. Such polynomials can be written in the form $\left(1-x^{2}\right) p_{n-2}(x)$ where $p_{n-2}(x)$ is an arbitrary polynomial of degree $n-2$, and the Galerkin equations can be written as

$$
\int_{-1}^{1} R_{n}(x)\left(1-x^{2}\right) p_{n-2}(x) W(x) d x=0 .
$$

For the Jacobi weight $W(x)=W_{\alpha, \beta}(x)=(1-x)^{\alpha}(1+x)^{\beta}$, the Galerkin method is therefore equivalent to the Tau method for the weight $W_{\alpha+1, \beta+1}(x)$ and the residual controlled by (1.7) has the form

$$
R_{n}(x)=\tau_{0} \lambda P_{n}^{(\alpha+1, \beta+1)}(x)+\tau_{1} \lambda P_{n-1}^{(\alpha+1, \beta+1)}(x) .
$$

This residual can be written in terms of the derivatives of $P_{n+1}^{(\alpha+1, \beta+1)}(x)$ and $P_{n}^{(\alpha, \beta)}(x)$ by making use of (B.7). Since we consider a range of parameters $\alpha$ and $\beta$, the JacobiTau method also includes some Jacobi-Galerkin methods.

In the collocation approach, $u_{n}(x)$ is determined from the boundary conditions $u_{n}( \pm 1)=0$ and enforcing $R_{n}\left(x_{j}\right)=0$ at the $n-1$ interior Gauss-Lobatto points $x_{j}$ such that $D P_{n}^{(\alpha, \beta)}\left(x_{j}\right)=0, j=1, \ldots, n-1$ [3, $\left.\S 2.2\right]$. The residual (1.3) takes the form [7] eqn. (4.5)]

$$
R_{n}(x)=(A+B x) D P_{n}^{(\alpha, \beta)}(x),
$$

for some $A$ and $B$ independent of $x$. The collocation residual (1.9) is provided for completeness since we do not have results about the collocation method and we raise doubts about the validity of the proof proposed in [7. That residual can be written in several equivalent forms by using the properties of Jacobi polynomials (sect. B.1).

The characteristic polynomials for the eigenvalues $\mu=1 / \lambda$ are derived in section 2 from the explicit expression (1.4) for $u_{n}(x)$ in terms of the residual $R_{n}(x)$ whose form is specified by the Jacobi Tau (or Galerkin) method as in (1.6) and (1.8). The zeros of these characteristic polynomials are shown to be real, negative and distinct in section 3 Recurrence relations for the Gegenbauer Tau characteristic polynomials are derived in section 4 where it is shown that the underlying fundamental matrix structure is tridiagonal + one row. Section [ [discusses some implementation issues and shows that the spectral integration implementation directly leads to the tridiagonal + one row structure which is well-conditioned. Some of the key properties of Jacobi and Gegenbauer polynomials used in this paper are summarized in appendix B We use a non-standard normalization for Gegenbauer polynomials, denoted $G_{n}^{(\gamma)}(x)$, since the standard normalization $C_{n}^{(\gamma)}(x)$ is singular in the Chebyshev case.

\section{Characteristic Polynomials.}

2.1. Jacobi-Tau method. Substituting (1.6) into (1.4), the Jacobi-Tau approximation can be written explicitly in terms of the yet undertermined constants $\tau_{0}, \tau_{1}$ 
and the eigenvalue $\mu=1 / \lambda$, as

$$
u_{n}(x)=\tau_{0} \sum_{k=0}^{\left[\frac{n}{2}\right]} \mu^{k} D^{2 k} P_{n}^{(\alpha, \beta)}(x)+\tau_{1} \sum_{k=0}^{\left[\frac{n-1}{2}\right]} \mu^{k} D^{2 k} P_{n-1}^{(\alpha, \beta)}(x) .
$$

The boundary conditions $u_{n}( \pm 1)=0$ then yield the characteristic equations

$$
\left\{\begin{array}{c}
\tau_{0} \sum_{k=0}^{\left[\frac{n}{2}\right]} \mu^{k} D^{2 k} P_{n}^{(\alpha, \beta)}(1)+\tau_{1} \sum_{k=0}^{\left[\frac{n-1}{2}\right]} \mu^{k} D^{2 k} P_{n-1}^{(\alpha, \beta)}(1)=0 \\
\tau_{0} \sum_{k=0}^{\left[\frac{n}{2}\right]} \mu^{k} D^{2 k} P_{n}^{(\alpha, \beta)}(-1)+\tau_{1} \sum_{k=0}^{\left[\frac{n-1}{2}\right]} \mu^{k} D^{2 k} P_{n-1}^{(\alpha, \beta)}(-1)=0
\end{array}\right.
$$

Equation (B.1) shows that $P_{n}^{(\alpha, \beta)}(-1)=(-1)^{n} P_{n}^{(\beta, \alpha)}(1)$ so the 2 nd equation above can be rewritten at $x=1$ by flipping the indices $\alpha$ and $\beta$,

$$
\left\{\begin{array}{l}
\tau_{0} \sum_{k=0}^{\left[\frac{n}{2}\right]} \mu^{k} D^{2 k} P_{n}^{(\alpha, \beta)}(1)+\tau_{1} \sum_{k=0}^{\left[\frac{n-1}{2}\right]} \mu^{k} D^{2 k} P_{n-1}^{(\alpha, \beta)}(1)=0 \\
\tau_{0} \sum_{k=0}^{\left[\frac{n}{2}\right]} \mu^{k} D^{2 k} P_{n}^{(\beta, \alpha)}(1)-\tau_{1} \sum_{k=0}^{\left[\frac{n-1}{2}\right]} \mu^{k} D^{2 k} P_{n-1}^{(\beta, \alpha)}(1)=0 .
\end{array}\right.
$$

This system has a non-trivial solution $\left(\tau_{0}, \tau_{1}\right) \neq(0,0)$ if and only if

$$
\begin{aligned}
\sum_{k=0}^{\left[\frac{n}{2}\right]} \mu^{k} D^{2 k} P_{n}^{(\alpha, \beta)}(1) \sum_{k=0}^{\left[\frac{n-1}{2}\right]} \mu^{k} & D^{2 k} P_{n-1}^{(\beta, \alpha)}(1) \\
& \quad+\sum_{k=0}^{\left[\frac{n-1}{2}\right]} \mu^{k} D^{2 k} P_{n-1}^{(\alpha, \beta)}(1) \sum_{k=0}^{\left[\frac{n}{2}\right]} \mu^{k} D^{2 k} P_{n}^{(\beta, \alpha)}(1)=0 .
\end{aligned}
$$

This is the characteristic equation for the eigenvalue $\mu$.

2.2. Gegenbauer-Tau method. Gegenbauer polynomials $G_{n}^{(\gamma)}(x)$ are the class of Jacobi polynomials $P_{n}^{(\alpha, \beta)}(x)$ with equal indices $\alpha=\beta=\gamma-1 / 2$ (sect. B.2). The Gegenbauer polynomials are even in $x$ for $n$ even and odd for $n$ odd [1, 22.4.2]. Chebyshev and Legendre polynomials are Gegenbauer polynomials with $\gamma=0$ and $1 / 2$, respectively. The symmetry of the differential equation (1.1) and of the Gegenbauer polynomials allows decoupling of the discrete problem into even and odd solutions. This parity reduction leads to simpler residuals and simpler forms for the corresponding characteristic polynomials. The residual in the parity-separated Gegenbauer case contains only one term

$$
R_{n}(x)=\tau_{0} \lambda G_{n}^{(\gamma)}(x)
$$

where $G_{n}^{(\gamma)}(x)$ is the $n^{\text {th }}$ Gegenbauer polynomial and $n$ is even for even solutions and odd for odd solutions. Substituting (2.5) into (1.4) provides the Gegenbauer-Tau 
approximation to (1.1) in terms of an undertermined constant $\tau_{0}$ and the eigenvalue $\mu=1 / \lambda$

$$
u_{n}(x)=\tau_{0} \sum_{k=0}^{\left[\frac{n}{2}\right]} \mu^{k} D^{2 k} G_{n}^{(\gamma)}(x) .
$$

The boundary condition $u_{n}( \pm 1)=0$ leads to the characteristic polynomial equation

$$
\sum_{k=0}^{\left[\frac{n}{2}\right]} \mu^{k} D^{2 k} G_{n}^{(\gamma)}(1)=0,
$$

since by symmetry $G_{n}^{(\gamma)}(-1)=(-1)^{n} G_{n}^{(\gamma)}(1)$ and the two boundary conditions give the same equation.

\section{Zeros of characteristic polynomials.}

3.1. Stable polynomials and the Hermite Biehler Theorem. The general approach to prove that the eigenvalues are real, negative and distinct is to construct a particular stable polynomial $p(z)$ then to use the Hermite Biehler theorem to deduce that the polynomials $\Omega_{1}(\mu)$ and $\Omega_{2}(\mu)$ such that $p(z)=\Omega_{1}\left(z^{2}\right)+z \Omega_{2}\left(z^{2}\right)$ have real, negative and distinct zeros that interlace.

Definition 3.1. A real polynomial, $p(z)$, is a stable polynomial (or a Hurwitz polynomial), if all its zeros lie in the open left half-plane, i.e. their real part is strictly less than zero, $\Re z<0$.

Definition 3.2. Let $\Omega_{1}(\mu)$ and $\Omega_{2}(\mu)$ be two real polynomials of degree $n$ and $n-1$ (or $n$ ) respectively, then $\Omega_{1}(\mu)$ and $\Omega_{2}(\mu)$ form a positive pair if: (a) the roots $\mu_{1}, \ldots, \mu_{n}$ of $\Omega_{1}$ and $\mu_{1}^{\prime} \cdots, \mu_{n-1}^{\prime}$ (or $\mu_{1}^{\prime}, \cdots, \mu_{n}^{\prime}$ ) of $\Omega_{2}$ are real, negative and distinct; (b) the roots strictly interlace (or alternate) as follows:

$$
\mu_{1}<\mu_{1}^{\prime}<\cdots<\mu_{n-1}^{\prime}<\mu_{n}<0 \quad\left(\text { or } \mu_{1}^{\prime}<\mu_{1}<\cdots<\mu_{n}^{\prime}<\mu_{n}<0\right) ;
$$

(c) the highest coefficients of $\Omega_{1}(\mu)$ and $\Omega_{2}(\mu)$ are of like sign.

We will use the following theorems about positive pairs, [14 p. 198], [17. Sec. 2]:

Lemma 3.3. Any nontrivial real linear combination of two polynomials that form a positive pair has real roots.

Lemma 3.4. Let $P(\mu)$ and $Q(\mu)$ be real standard polynomials (i.e. the leading coefficient is positive) with only non-positive zeros. Then $P(\mu)$ interlaces (or alternates) $Q(\mu)$ (in the sense of definition [3.2, but not strictly) if and only if for all $A>0$ both $Q(\mu)+A P(\mu)$ and $Q(\mu)+A \mu P(\mu)$ have only non positive zeros.

The polynomials $P$ and $Q$ of theorem 3.4 do not necessarily form a positive pair since they are allowed to have common and/or multiple roots. We call this set of polynomials a quasi-positive pair.

Lemma 3.5. [7. Lemma 3.4] If $\Omega_{1}(\mu), \Omega_{2}(\mu)$ and $\Theta_{1}(\mu), \Theta_{2}(\mu)$ are two positive pairs then the zeros of $H(\mu)=\Omega_{1}(\mu) \Theta_{2}(\mu)+\Omega_{2}(\mu) \Theta_{1}(\mu)$ are real, negative and distinct.

Stability (definition 3.1) is very important in temporal discretizations and matrix theory [3] as well as in analysis (e.g. [10] and references therein). Stable polynomials can surface as characteristic polynomials of a numerical method applied on a differential equation. A necessary and sufficient condition for a polynomial to be stable is given by the Routh-Hurwitz theorem (see, for example, [12, §40], [13, §23]). Other important characterizations of stable polynomials are the Routh-Hurwitz criterion and 
the total positivity of a Hurwitz matrix [10, although these will not be used here. The characterization of stable polynomials that will be most useful here is given by the Hermite-Biehler Theorem [14 p. 197], [10].

THEOREM 3.6 (Hermite-Biehler). The polynomial with real coefficients $p(z)=$ $\Omega_{1}\left(z^{2}\right)+z \Omega_{2}\left(z^{2}\right)$ is stable, if and only if $\Omega_{1}(\mu)$ and $\Omega_{2}(\mu)$ form a positive pair.

The Hermite Biehler theorem states that the even and odd parts of stable polynomials form positive pairs. This supplies us with a very strong tool to prove reality and negativity of the roots of certain polynomials.

Gottlieb and Lustman [7] used the Hermite Biehler theorem to prove that the spectrum of the Chebyshev collocation operator for the heat equation is real, negative and distinct for a variety of homogeneous boundary conditions. The basic strategy is to show that the characteristic polynomial for that method are the even or odd parts of a stable polynomial. Our results extend their strategy to a class of Jacobi and Gegenbauer Tau methods that includes Chebyshev and Legendre Tau and Galerkin formulations. Although the general approach is similar to that of Gottlieb [6] and Gottlieb and Lustman [7, the extension is technically non-trivial and there are differences and some corrections. The key steps in [7] is to prove that the polynomials [7. (4.11),(4.12)] are stable. To do so, Gottlieb and Lustman derive a first order differential equation for those polynomials then transform that ODE into an inhomogeneous one-way wave equation [7, (4.13)] and call on the results [6, (3.18),(3.20)] to deduce stability. This is not quite correct since the eigenvalue $\mu$ here is complex hence $w_{N}(x, t)$ in [7. (4.13)] is also complex while Gottlieb implicitly assumes reality of $v_{N}(x, t)$ and $R_{N}(x, t)$ in [6] (3.18),(3.20)].

The proof for [7. (4.11)] can be fixed and generalized as done in [4] (theorem 3.7 below) where we deduce stability of the polynomials (3.1) below without going back

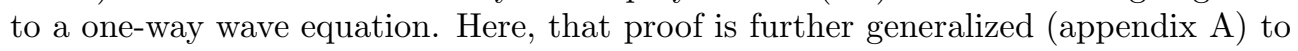
the polynomials (3.2) and (3.3) below in order to prove our results about the Jacobi Tau method for the 2nd order operator. Our proof follows Gottlieb's ideas to derive the results [6] (3.18), (3.20)] although we do not use Gauss integration.

The proof for [7. (4.12)] does not appear to be correct however and we have not succeeded in obtaining a corrected proof. Gottlieb and Lustman do not provide a proof of stability for that polynomial (4.12), they state only that a 'similar argument holds'. Gottlieb [6] likewise suggests that the proof of stability for [6] (3.11b)] implies stability for [6. (3.11a)] but this is not evident since $\tau_{1}(t)$ and $\tau_{2}(t)$ are distinct functions of time that are fully determined by their respective solution procedure. Gottlieb also suggests that $v_{N}(x, t)[6,(3.8)]$ is directly related to $u_{N}(x, t)[6$, $(3.2)]$ by relation [6] (3.8)]. It is true that $T_{N}\left(x_{n}\right)$ can be eliminated for $n=0, \ldots, N-1$ as suggested in the derivation of [6] (3.8)], since $(1-x) D T_{N}(x)=2(-1)^{N-1} \sum_{k=0}^{N}(-1)^{k} c_{k}^{-1} T_{k}(x)$ as can be deduced from [6 (3.2),(3.3)]. However this does not imply that the resulting $d_{k}$ coefficients [6. (3.8)] deduced from the $a_{k}$ 's that solve [6. (3.6)] are the same $d_{k}$ 's as those that solve [6] (3.10)].

Hence, it appears that there is currently no proof of the stability of [6. (3.6),(3.11a)] and [7. (4.12)], therefore invalidating Gottlieb and Lustman's proof that the eigenvalues of the Chebyshev collocation operator are real, negative and distinct [7].

3.2. Important Stable Polynomials and Positive Pairs. Here we prove stability of certain real polynomials whose even and odd parts are directly related to the characteristic polynomials derived in section 2 for the Jacobi Tau method. 
TheOREM 3.7. Let $P_{n}^{(\alpha, \beta)}(x)$ denote the Jacobi polynomial of degree $n$, where $n \geq 2$. If $-1<\alpha \leq 1$ and $\beta>-1$, then the zeros of the polynomial

$$
\Phi_{n}(\mu):=\sum_{k=0}^{n}\left(\frac{d^{k}}{d x^{k}} P_{n}^{(\alpha, \beta)}(x)\right)_{x=1} \mu^{k}
$$

lie in the left half-plane; that is, $\Phi_{n}(\mu)$ is a stable polynomial. The proof of this theorem is in [4] together with a discussion of its relation to zeros of Bessel polynomials. The next two theorems give two generalizations of the above result that are needed for this paper.

THEOREM 3.8. Let $P_{n}^{(\alpha, \beta)}(x)$ denote the Jacobi polynomial of degree $n$, with $n \geq 3$, then the polynomial

$$
\Phi_{n}^{1}(\mu):=\sum_{k=0}^{n}\left(\frac{d^{k}}{d x^{k}} P_{n}^{(\alpha, \beta)}(x)\right)_{x=1} \mu^{k}+A \sum_{k=0}^{n-1}\left(\frac{d^{k}}{d x^{k}} P_{n-1}^{(\alpha, \beta)}(x)\right)_{x=1} \mu^{k}
$$

is stable for every $A \geq 0$ when $-1<\alpha \leq 0$ and $\beta>-1$.

THEOREM 3.9. Let $P_{n}^{(\alpha, \beta)}(x)$ denote the Jacobi polynomial of degree $n$, with $n \geq 3$, then the polynomial

$$
\Phi_{n}^{2}(\mu):=\sum_{k=0}^{n}\left(\frac{d^{k}}{d x^{k}} P_{n}^{(\alpha, \beta)}(x)\right)_{x=1} \mu^{k}+A \mu^{2} \sum_{k=0}^{n-1}\left(\frac{d^{k}}{d x^{k}} P_{n-1}^{(\alpha, \beta)}(x)\right)_{x=1} \mu^{k}
$$

is stable for every $A \geq 0$ when $-1<\alpha \leq 1$ and $\beta>-1$.

The proofs of these theorems are technical and they are given in appendix $\mathrm{A}$ Our next theorem combines all the above theorems to get an important result.

Theorem 3.10. Let $n \geq 3$, then the polynomials

$$
\Omega_{n}^{(\alpha, \beta)}(\mu):=\sum_{k=0}^{\left[\frac{n}{2}\right]} \mu^{k} D^{2 k} P_{n}^{(\alpha, \beta)}(1), \quad \Omega_{n-1}^{(\alpha, \beta)}(\mu):=\sum_{k=0}^{\left[\frac{n-1}{2}\right]} \mu^{k} D^{2 k} P_{n-1}^{(\alpha, \beta)}(1)
$$

form a positive pair if $-1<\alpha, \beta \leq 0$ or $0<\alpha, \beta \leq 1$.

REMARK 1. It was shown in [4] that these polynomials have real negative and distinct roots for $-1<\alpha \leq 1$ and $-1<\beta$. The important addition of theorem 3.10 is that the roots of these polynomials interlace as was conjectured in [4].

Proof. Applying the Hermite-Biehler Theorem to the stable polynomials of theorem 3.7 for a given $n$ and also for $n-1 \geq 2$, proves that the polynomials $\Omega_{n}^{(\alpha, \beta)}(\mu)$ and $\Omega_{n-1}^{(\alpha, \beta)}(\mu)$ have real negative and distinct roots for $-1<\alpha, \beta \leq 1$ (Notice that we can interchange $\alpha$ and $\beta$ ). Applying the Hermite-Biehler Theorem to the stable polynomials of theorems 3.8 and 3.9 shows that the polynomials

$$
\begin{aligned}
& \sum_{k=0}^{\left[\frac{n}{2}\right]} \mu^{k} D^{2 k} P_{n}^{(\alpha, \beta)}(1)+A \sum_{k=0}^{\left[\frac{n-1}{2}\right]} \mu^{k} D^{2 k} P_{n-1}^{(\alpha, \beta)}(1)=\Omega_{n}^{(\alpha, \beta)}(\mu)+A \Omega_{n-1}^{(\alpha, \beta)}(\mu), \\
& \sum_{k=0}^{\left[\frac{n}{2}\right]} \mu^{k} D^{2 k} P_{n}^{(\alpha, \beta)}(1)+A \mu \sum_{k=0}^{\left[\frac{n-1}{2}\right]} \mu^{k} D^{2 k} P_{n-1}^{(\alpha, \beta)}(1)=\Omega_{n}^{(\alpha, \beta)}(\mu)+A \mu \Omega_{n-1}^{(\alpha, \beta)}(\mu)
\end{aligned}
$$

have real negative and distinct roots for all $A>0$ and $-1<\alpha, \beta \leq 0$. These results provide sufficient information to apply lemma 3.4 to deduce that the set of polynomials $\left(\Omega_{n}^{(\alpha, \beta)}(\mu), \Omega_{n-1}^{(\alpha, \beta)}(\mu)\right)$ form quasi-positive pair for $-1<\alpha, \beta \leq 0$. 
To show that these polynomials form a positive pair recall that both $\Omega_{n}^{(\alpha, \beta)}(\mu)$ and $\Omega_{n-1}^{(\alpha, \beta)}(\mu)$ have real, negative and distinct roots by theorem 3.7 Thus it remains to show that they have no common roots. To do so, assume that $\mu$ is a common root. Then $P_{1}(\mu)=\Omega_{n}^{(\alpha, \beta)}(\mu)+A \Omega_{n-1}^{(\alpha, \beta)}(\mu)=0$ and $P_{2}(\mu)=\Omega_{n}^{(\alpha, \beta)}(\mu)+A \mu \Omega_{n-1}^{(\alpha, \beta)}(\mu)=0$. Since $D \Omega_{n}^{(\alpha, \beta)}(\mu) \neq 0$ and $D \Omega_{n-1}^{(\alpha, \beta)}(\mu) \neq 0$ (both $\Omega_{n}$ and $\Omega_{n-1}$ do not have a double root), set $A=-\frac{D \Omega_{n}^{(\alpha, \beta)}(\mu)}{D \Omega_{n-1}^{(\alpha, \beta)}(\mu)}$ or $A=-\frac{D \Omega_{n}^{(\alpha, \beta)}(\mu)}{\mu D \Omega_{n-1}^{(\alpha, \beta)}(\mu)}$, whichever one is positive (one of the two must be since $\mu$ is negative). But this will imply that $D P_{1}(\mu)=0$ and $D P_{2}(\mu)=0$ respectively, a contradiction since $P_{1}(\mu)$ and $P_{2}(\mu)$ have simple zeros. For the second range of parameters, replace $n$ with $n+1$ in theorems 3.8 and 3.9 and apply the Hermite-Biehler Theorem. This gives that the polynomials

$$
\begin{aligned}
& \sum_{k=0}^{\left[\frac{n}{2}\right]} \mu^{k} D^{2 k+1} P_{n+1}^{(\alpha, \beta)}(1)+A \sum_{k=0}^{\left[\frac{n-1}{2}\right]} \mu^{k} D^{2 k+1} P_{n}^{(\alpha, \beta)}(1) \\
& \sum_{k=0}^{\left[\frac{n}{2}\right]} \mu^{k} D^{2 k+1} P_{n+1}^{(\alpha, \beta)}(1)+A \mu \sum_{k=0}^{\left[\frac{n-1}{2}\right]} \mu^{k} D^{2 k+1} P_{n}^{(\alpha, \beta)}(1)
\end{aligned}
$$

also have real negative and distinct roots. Using (B.7) these polynomials transform to

$$
\begin{aligned}
& \sum_{k=0}^{\left[\frac{n}{2}\right]} \mu^{k} D^{2 k} P_{n}^{(\alpha+1, \beta+1)}(1)+A^{\prime} \sum_{k=0}^{\left[\frac{n-1}{2}\right]} \mu^{k} D^{2 k} P_{n-1}^{(\alpha+1, \beta+1)}(1) \\
& \sum_{k=0}^{\left[\frac{n}{2}\right]} \mu^{k} D^{2 k} P_{n}^{(\alpha+1, \beta+1)}(1)+A^{\prime} \mu \sum_{k=0}^{\left[\frac{n-1}{2}\right]} \mu^{k} D^{2 k} P_{n-1}^{(\alpha+1, \beta+1)}(1) .
\end{aligned}
$$

The new polynomials have real negative and distinct roots for all $A^{\prime}>0$, where $A^{\prime}=\frac{n+\alpha+\beta}{n+1+\alpha+\beta} A$, and thus the second range $0<\alpha, \beta \leq 1$ follows from the first one by a simple change of variables. $\square$

3.3. Eigenvalues of the Gegenbauer and Jacobi Tau methods. The previous subsection provides all necessary information needed for proving reality and negativity of the eigenvalues. First consider the Gegenbauer case.

THEOREM 3.11. The eigenvalues of the Gegenbauer Tau discretization of the second order operator with Dirichlet boundary conditions, problem [1.1, are real negative and distinct for $-1 / 2<\gamma \leq 5 / 2$. Also, characteristic polynomials given by successive order (i.e. $n-1$ and $n$ ) approximations interlace.

Proof. The eigenvalues are the roots of (2.7). The theorem follows directly from theorem 3.10] since $\alpha=\beta=\gamma-1 / 2$ in the Gegenbauer case and the two ranges for the indices $(\alpha, \beta)$ merge into the single range $-1 / 2<\gamma \leq 5 / 2$.

REMARK 2. The results of theorem 3.11 are sharp in the sense that well-conditioned numerical calculations (sect. 5.2) give some complex conjugate pairs of eigenvalues for $\gamma>5 / 2$ and Gegenbauer integration (B.10) diverges in general for $\gamma \leq-1 / 2$.

REMARK 3. As pointed out in the introduction, the Galerkin method with weight function $W_{\alpha, \beta}(x)$ for problem 1.1 is equivalent to the Tau method with weight function $W_{\alpha+1, \beta+1}(x)$. For the Gegenbauer case the Galerkin method with weight function $W_{\gamma}(x)$ is equivalent to the Tau method with weight function $W_{\gamma+1}(x)$. Thus, a direct consequence of theorem 3.11 is that the eigenvalues of the Gegenbauer Galerkin method 
are real negative and distinct for $-1 / 2<\gamma \leq 3 / 2$. Again, characteristic polynomials given by successive order approximations interlace.

REMARK 4. Theorem 3.11 includes the Chebyshev and Legendre polynomials. For $\gamma=0$, we have $G_{n}^{(0)}(x)=\frac{T_{n}(x)}{n}$, where $T_{n}(x)$ denotes the $n^{\text {th }}$ Chebyshev polynomial of the first kind [14, p. 19]. Thus the theorem implies that the Chebyshev-Tau method has real negative and distinct eigenvalues with interlacing characteristic polynomials given by successive order approximations. For $\gamma=\frac{1}{2}$ we have $G_{n}^{(1 / 2)}(x)=P_{n}(x)$, where $P_{n}(x)$ is the $n^{\text {th }}$ Legendre polynomial and for $\gamma=1$ we have that $G_{n}^{(1)}(x)=\frac{U_{n}(x)}{2}$ where $U_{n}(x)$ the $n^{\text {th }}$ Chebyshev polynomial of second kind. Therefore, the same result holds for both the Legendre Tau and the Chebyshev Tau of 2nd kind method. Furthermore, in the Galerkin case (see remark 3) the Galerkin Chebyshev, the Galerkin Legendre and the Galerkin Chebyshev of the 2nd kind methods have real, negative and distinct eigenvalues as well.

For the Jacobi case we have

THEOREM 3.12. The eigenvalues of the Jacobi Tau discretization of the second order operator with Dirichlet boundary conditions, problem [1.1] are real negative and distinct if $-1<\alpha, \beta \leq 0$ or $0<\alpha, \beta \leq 1$.

Proof. By theorem 3.10 the polynomials $\left(\Omega_{n}^{(\alpha, \beta)}(\mu), \Omega_{n-1}^{(\alpha, \beta)}(\mu)\right)$ form a positive pair for $-1<\alpha, \beta \leq 0$ and $0<\alpha, \beta \leq 1$. Interchanging the indices $\alpha$, $\beta$, the same result holds for $\left(\Omega_{n}^{(\beta, \alpha)}(\mu), \Omega_{n-1}^{(\beta, \alpha)}(\mu)\right)$. Application of theorem 3.5 to these sets of positive pairs gives that the polynomial

$$
B_{n}^{(\alpha, \beta)}(\mu)=\Omega_{n}^{(\alpha, \beta)}(\mu) \Omega_{n-1}^{(\beta, \alpha)}(\mu)+\Omega_{n}^{(\beta, \alpha)}(\mu) \Omega_{n-1}^{(\alpha, \beta)}(\mu)
$$

has real negative and distinct roots. Equation (2.4) shows that $B_{n}^{(\alpha, \beta)}(\mu)$ is the characteristic polynomial for the Jacobi Tau method. $\square$

This paper focuses on the second order problem with Dirichlet boundary conditions. Naturally, questions arise about the equivalent results for different boundary conditions. The next remarks gives some answer to that question.

REMARK 5. Consider problem [1.1] with Neumann boundary conditions i.e. $\lambda u-$ $D^{2} u=0$ with $D u( \pm 1)=0$. The Jacobi Tau method gives $\lambda u-D^{2} u=\tau_{0} P_{n}^{(\alpha, \beta)}(x)+$ $\tau_{1} P_{n-1}^{(\alpha, \beta)}(x)$. Notice that $\lambda=0$ is an eigenvalue since $u=$ constant is a solution. For $\lambda \neq 0$, differentiate the last equation to get $\lambda D u-D^{3} u=\tau_{0} D P_{n}^{(\alpha, \beta)}(x)+\tau_{1} D P_{n-1}^{(\alpha, \beta)}(x)$ with $D u( \pm 1)=0$. Set now $v=D u$ and with the use of B.7 the equation transforms to $\lambda v-D^{2} v=\tau_{0}^{\prime} P_{n-1}^{(\alpha+1, \beta+1)}(x)+\tau_{1}^{\prime} P_{n-2}^{(\alpha+1, \beta+1)}(x)$ with $v( \pm 1)=0$. This is the Jacobi Tau approximation of second kind i.e. $(\alpha, \beta) \rightarrow(\alpha+1, \beta+1)$ for problem [1.1] and by theorem 3.12 it has real negative and distinct eigenvalues for $-1<\alpha, \beta \leq 0$.

REMARK 6. Consider problem [1.1 with boundary conditions $u(-1)=0$ and $D u(1)=0$. Using equation 2.1 and following the ideas of subsection 2.1] we get that the characteristic polynomial in this case is $B_{n}^{(\alpha, \beta)}(\mu)=k_{n-1} \Omega_{n}^{(\beta, \alpha)}(\mu) \Omega_{n-2}^{(\alpha+1, \beta+1)}(\mu)+$ $k_{n} \Omega_{n-1}^{(\beta, \alpha)}(\mu) \Omega_{n-1}^{(\alpha+1, \beta+1)}(\mu)$, where $k_{n}=\frac{1}{2}(n+\alpha+\beta+1)$. Since the polynomials $\left(\Omega_{n}^{(\beta, \alpha)}(\mu), \Omega_{n-1}^{(\beta, \alpha)}\right)$ and $\left(k_{n} \Omega_{n-1}^{(\alpha+1, \beta+1)}(\mu), k_{n-1} \Omega_{n-2}^{(\alpha+1, \beta+1)}\right)$ form positive pairs for $-1<$ $\alpha, \beta \leq 0$ then by theorem 3.5 the roots of $B_{n}^{(\alpha, \beta)}(\mu)$ are real negative and distinct for $-1<\alpha, \beta \leq 0$.

An alternative implementation is to rescale the domain to $[-1,0]$ with the Neuman boundary condition $D u(0)=0$ and to use only the even Gegenbauer polynomials with a Gegenbauer-Tau approach on the entire domain $[-1,1]$ and boundary conditions $u( \pm 1)=0$ since such polynomials automatically satisfy $D u(0)=0$. 
4. Characteristic polynomial recurrences. The previous section shows that the characteristic polynomials given by successive order approximations of the Gegenbauer Tau method have real negative and distinct roots that interlace strictly, provided that $-1 / 2<\gamma \leq 5 / 2$. Reality of the roots as well as the interlacing property is an important characteristic of orthogonal polynomials of successive order [15], [14, p. 16]. These properties are direct consequences of the three term recurrence relations satisfied by orthogonal polynomials [15, 14]. Here, we derive recurrence relations for the characteristic polynomials of the Gegenbauer Tau method and show that they consist of three term recurrences plus a constant term in general. The constant term vanishes when $\gamma=1 / 2$ or $3 / 2$.

From (2.7), the characteristic polynomials for Gegenbauer-Tau approximations to the even and odd modes of (1.1) are, respectively,

$$
p_{m}(\mu):=\sum_{k=0}^{m} \mu^{k} D^{2 k} G_{2 m}^{(\gamma)}(1), \quad \text { and } \quad q_{m}(\mu):=\sum_{k=0}^{m} \mu^{k} D^{2 k} G_{2 m+1}^{(\gamma)}(1) .
$$

theorem 3.11 states that these polynomials have real, negative and distinct zeros for $-1 / 2<\gamma \leq 5 / 2$ and that the zeros of $p_{m}(\mu)$ and $q_{m}(\mu)$ interlace, as do the zeros of $q_{m-1}(\mu)$ and $p_{m}(\mu)$. Recurrence relations for these characteristic polynomials follow directly from recurrences for $2 \mathrm{nd}$ derivatives of Gegenbauer polynomials which, using (B.16) twice, read

$$
\left\{\begin{array}{l}
G_{0}^{(\gamma)}=\frac{D^{2} G_{2}^{(\gamma)}}{2(\gamma+1)}, \quad G_{1}^{(\gamma)}=\frac{D^{2} G_{3}^{(\gamma)}}{4(\gamma+1)(\gamma+2)}, \\
G_{n}^{(\gamma)}= \\
\frac{D^{2} G_{n+2}^{(\gamma)}}{4(\gamma+n+1)(\gamma+n)}-\frac{D^{2} G_{n}^{(\gamma)}}{2(\gamma+n+1)(\gamma+n-1)}+\frac{D^{2} G_{n-2}^{(\gamma)}}{4(\gamma+n)(\gamma+n-1)}
\end{array}\right.
$$

4.1. Recurrences for even modes. Substituting (4.2) with $n=2 m$ into the characteristic polynomials $p_{m}(\mu)$ defined in (4.1), with $p_{0}(\mu)=1$ since $G_{0}^{(\gamma)}:=1$, yields the recurrence

$$
\left\{\begin{array}{l}
\mu p_{0}(\mu)=\frac{p_{1}(\mu)}{2(\gamma+1)}-K_{0}^{(\gamma)} \\
\mu p_{1}(\mu)=\frac{p_{2}(\mu)}{4(\gamma+2)(\gamma+3)}-\frac{p_{1}(\mu)}{2(\gamma+1)(\gamma+3)}-K_{2}^{(\gamma)} \\
\mu p_{m}(\mu)= \\
\frac{p_{m+1}(\mu)}{4(\gamma+n+1)(\gamma+n)}-\frac{p_{m}(\mu)}{2(\gamma+n+1)(\gamma+n-1)}+\frac{p_{m-1}(\mu)}{4(\gamma+n)(\gamma+n-1)}-K_{n}^{(\gamma)}
\end{array}\right.
$$

where, using (B.17),

$$
\begin{aligned}
K_{0}^{(\gamma)} & :=\frac{G_{2}^{(\gamma)}(1)}{2(\gamma+1)}=\frac{2 \gamma+1}{4(\gamma+1)} \\
K_{2}^{(\gamma)} & :=\frac{G_{4}^{(\gamma)}(1)}{4(\gamma+2)(\gamma+3)}-\frac{G_{2}^{(\gamma)}(1)}{2(\gamma+1)(\gamma+3)}=\frac{\left(2 \gamma^{2}+\gamma-7\right)(1+2 \gamma)}{48(\gamma+1)(\gamma+2)} .
\end{aligned}
$$

and

$$
K_{n}^{(\gamma)}=\frac{G_{n+2}^{(\gamma)}(1)}{4(\gamma+n+1)(\gamma+n)}-\frac{G_{n}^{(\gamma)}(1)}{2(\gamma+n+1)(\gamma+n-1)}+\frac{G_{n-2}^{(\gamma)}(1)}{4(\gamma+n)(\gamma+n-1)}
$$


for $n \geq 3$. From (B.17), the latter expression reduces to

$$
K_{n}^{(\gamma)}=\frac{(2 \gamma-1)(2 \gamma-3)}{n\left(n^{2}-1\right)(n+2)} G_{n-2}^{(\gamma)}(1)=\frac{(2 \gamma-1)(2 \gamma-3)}{n\left(n^{2}-1\right)\left(n^{2}-4\right)}\left(\begin{array}{c}
2 \gamma+n-3 \\
n-3
\end{array}\right),
$$

for $n \geq 3$, and obeys the recurrence

$$
K_{n+2}^{(\gamma)}=\frac{(2 \gamma+n-1)(2 \gamma+n-2)}{(n+4)(n+3)} K_{n}^{(\gamma)} \quad \text { with } \quad K_{4}^{(\gamma)}=\frac{\left(4 \gamma^{2}-1\right)(2 \gamma-3)}{720} .
$$

4.2. Recurrences for odd modes. Substituting (4.2) with $n=2 m+1$ into the characteristic polynomials for the odd modes $q_{m}(\mu)$ defined in (4.1), with $q_{0}(\mu)=1$, gives

$$
\left\{\begin{array}{l}
\mu q_{0}(\mu)=\frac{q_{1}(\mu)}{4(\gamma+1)(\gamma+2)}-K_{1}^{(\gamma)} \\
\mu q_{m}(\mu)= \\
\frac{q_{m+1}(\mu)}{4(\gamma+n+1)(\gamma+n)}-\frac{q_{m}(\mu)}{2(\gamma+n+1)(\gamma+n-1)}+\frac{q_{m-1}(\mu)}{4(\gamma+n)(\gamma+n-1)}-K_{n}^{(\gamma)}
\end{array}\right.
$$

where, using (B.17), $K_{1}^{(\gamma)}:=[4(\gamma+1)(\gamma+2)]^{-1} G_{3}^{(\gamma)}(1)=[12(\gamma+2)]^{-1}(2 \gamma+1)$ and $K_{n}^{(\gamma)}$ as in (4.5) but here with $n=2 m+1$. The recurrence (4.7) applies here also but starting now with $K_{3}^{(\gamma)}=(2 \gamma-1)(2 \gamma-3) / 120$.

In general, (4.3) and (4.8) are three term recurrences plus the constants $K_{n}^{(\gamma)}$. These constants vanish for all $n \geq 3$ when $\gamma=1 / 2$, the Tau-Legendre method, and when $\gamma=3 / 2$, the Tau-Legendre of the 2nd kind or Galerkin-Legendre method. In those cases, the recurrences have only three terms hence the corresponding $p_{m}(\mu)$ and $q_{m}(\mu)$ sequences of polynomials are orthogonal polynomials [14 p. 13 and references therein]. The recurrence (4.7) for $K_{n}^{(\gamma)}$ also indicates why $\gamma=5 / 2$ is a critical value in theorem 3.11 For $\gamma<5 / 2,(2 \gamma+n-1)(2 \gamma+n-2)<(n+4)(n+3)$ and $K_{n}^{(\gamma)}$ decreases with increasing $n$, while for $\gamma>5 / 2, K_{n}^{(\gamma)}$ increases with $n$. If $K_{n}^{(\gamma)}=0$ for $n \geq 3$, the characteristic polynomial sequences $p_{m}(\mu)$ and $q_{m}(\mu)$ satisfy a three term recurrence, respectively, therefore they are orthogonal and have real roots that interlace. The constants $K_{n}^{(\gamma)} \neq 0$ pulls down or pushes up the successive polynomials in the sequences with respect to that orthogonal case. For $\gamma>5 / 2$ that shift leads to the bifurcation from real eigenvalues to complex conjugate pairs.

\section{Numerical Implementation.}

5.1. Matrix formulation of the recurrences. The recurrences (4.3) and (4.8) for the characteristic polynomials can be expressed in the matrix form

$$
\mu\left[p_{0}(\mu), p_{1}(\mu), \cdots\right]=\left[p_{0}(\mu), p_{1}(\mu), \cdots\right] M
$$

where the semi-infinite matrix $M$ is tridiagonal plus one row. The matrix $M$ is purely tridiagonal if $\gamma=1 / 2$ or $3 / 2$. The roots of the $m$-th order polynomial $p_{m}(\mu)$ are the eigenvalues of the $m$-by- $m$ matrix $M(0: m-1,0: m-1)$. A matlab code, buildGI2.m, is provided in appendix $[$ which constructs the matrix $\mathrm{GI} 2=M(0: m, 0: m-1)$ for both the even and odd modes by direct implementation of formulas (4.3) and (4.8) with (4.7). This approach provides an effective and well-conditioned technique to compute the Gegenbauer-Tau eigenvalues as illustrated in figure 5.1] which shows the 
odd mode eigenvalues for two values of $m \equiv$ MG, the total number of modes, for $\gamma=0$, $0.5,1,1.5$, corresponding to Chebyshev-Tau, Legendre-Tau, Chebyshev-Galerkin and Legendre-Tau, respectively. The Gegenbauer-Tau approximations involve an expansion of the solution $u_{n}(x)$ into odd polynomials up to degree $n=2 \mathrm{MG}+1$ and therefore up to degree 2001 for $\mathrm{MG}=1000$. That calculation shows that slightly more than $60 \%$ of the spectrum is captured with close to machine precision (here double precision IEEE arithmetic), demonstrating the excellent numerical conditioning of the formulation. Comparing the $\mathbf{M G}=100$ and $\mathbf{M G}=1000$ calculations shows that there is a slight ballooning of the round-off error at the higher truncation level. This might be explained by assuming randomness of roundoff errors with a standard deviation growing like $\sqrt{\mathrm{MG}}$. It would be interesting to obtain asymptotic estimates for the high frequency modes, $k>0.6$ MG. The largest eigenvalues for $\mathrm{MG}=1000$ and $\gamma=0,0.5,1$, and 1.5 are, respectively, $4.86 \times 10^{12}, 1.63 \times 10^{12}, 7.61 \times 10^{11}$ and $4.07 \times 10^{11}$. So the Legendre-Galerkin method can be said to be slightly less stiff than the other methods. These values are consistent with estimates that the largest eigenvalues are $O\left(\mathrm{MG}^{4}\right)[18$.
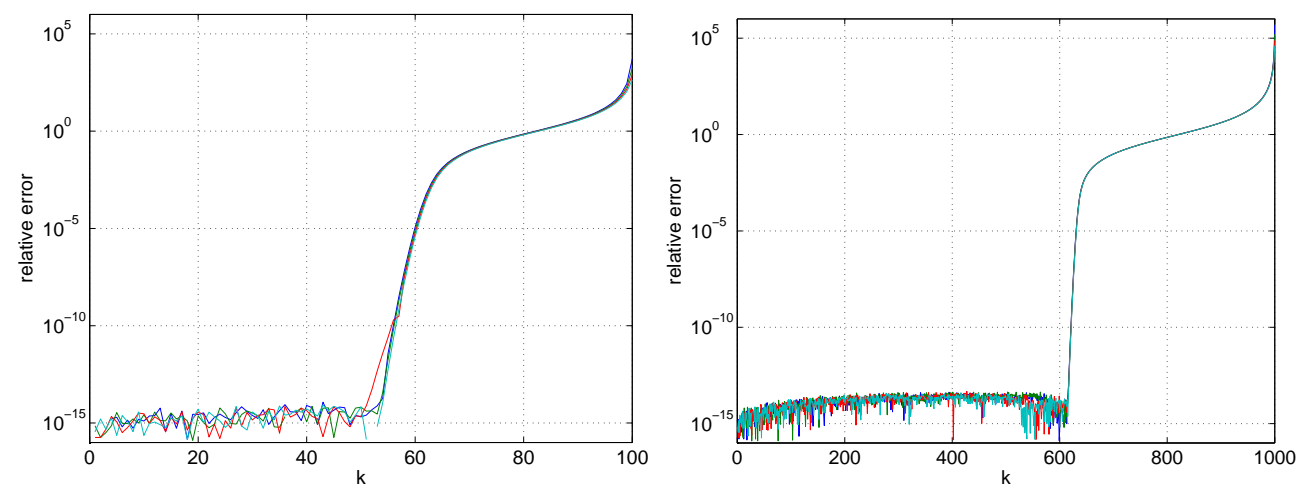

FIG. 5.1. Relative error $\left|\lambda-\lambda_{e}\right| /\left|\lambda_{e}\right|$ for the entire odd mode spectrum for $M G=100$ (left) and $M G=1000$ (right). Exact eigenvalues are $\lambda_{e}=-k^{2} \pi^{2}, k=1, \ldots, M G$. The relative errors for $\gamma=0$, 0.5, 1 and 1.5 are shown but essentially indistinguishable at this scale.

5.2. Don't Differentiate, Integrate. Our approach so far has been theoretical and focused on the basic eigenproblem (1.1). For more general two-point boundary value problems, e.g. nonlinear problems, it would not be possible to obtain explicit forms such as (1.4) for the discrete solution, and the residuals would not be as simple as (1.6) or 2.5]. For more general applications it is necessary to select explicit bases for the trial and test functions and to perfom the integrals (1.5) or (1.7) by Gauss integration.

One classical implementation of the Gegenbauer-Tau method is to expand $u_{n}(x)$ in terms of Gegenbauer polynomials $u_{n}(x)=\sum_{l=0}^{n} a_{l} G_{l}^{(\gamma)}(x)$ and to use the $n-1$ Gegenbauer polynomials $G_{k}^{(\gamma)}(x), k=0, \ldots, n-2$ as the test functions in lieu of $p_{n-2}(x)$ in 1.5. Those $n-1$ integrals, computed by Gauss quadrature in practice, and the two boundary conditions yield the $n+1$ equations to determine the $n+1$ coefficients $a_{k}$. For the even modes of the simple eigenproblem (1.1), this formulation consists of the even expansion

$$
u_{2 m}(x)=\sum_{l=0}^{m} a_{l} G_{2 l}^{(\gamma)}(x) \quad \Rightarrow \quad D^{2} u_{2 m}(x)=\sum_{l=0}^{m} a_{l} D^{2} G_{2 l}^{(\gamma)}(x)
$$


with the weighted residual equations

$$
\int_{-1}^{1}\left(D^{2} u_{2 m}-\lambda u_{2 m}\right) G_{2 k}^{(\gamma)} W^{(\gamma)} d x=0, \quad k=0, \ldots, m-1
$$

where $W^{(\gamma)}=\left(1-x^{2}\right)^{\gamma-1 / 2}$. Equations (5.3) yield a matrix problem $A a=\lambda B a$ for $a=\left[a_{0}, \ldots, a_{m}\right]^{T}$, where the $m$-by- $(m+1)$ matrix $B$ is diagonal plus one zero column, from orthogonality of the Gegenbauer polynomials $(\mathbf{B} .10)$, and the $m$-by- $(m+1)$ matrix $A$ is upper triangular with $A(k, l)=0$ for $k \geq l$ since, from (4.2), $D^{2} G_{2 l}(x)$ can be expressed in terms of all the Gegenbauer polynomials of even degree less than $2 l$. The boundary condition $u_{n}(1)=\sum_{l=0}^{m} a_{l} G_{2 l}^{(\gamma)}(1)=0$ allows the elimination of one of the coefficients, $a_{0}$ or $a_{m}$ say. This elimination can be expressed in the form $a=C \tilde{a}$ where $\tilde{a}$ is the column vector containing the remaining $m$ coefficients and $C$ is an $(m+1)$-by- $m$ matrix consisting of the $m$-by- $m$ identity matrix plus one full row. This yields the generalized eigenvalue problem $A C \tilde{a}=\lambda B C \tilde{a}$. The structure of the resulting matrices $(A C)$ and $(B C)$ depends on which coefficient is eliminated. If $a_{m}$ is eliminated, then $(A C)$ is full and $(B C)$ is diagonal. If $a_{0}$ is eliminated then $(A C)$ is upper triangular and $(B C)$ is zero everywhere except on the first row and the first lower diagonal.

Many other implementations are possible. For instance, one can use a polynomial expansion that satisfies the boundary conditions a priori, $u_{2 m}(x)=(1-$ $\left.x^{2}\right) \sum_{l=0}^{m-1} b_{l} \varphi_{2 l}(x)$, where $\varphi_{2 l}(x)$ is an even polynomial of degree $2 l$. Picking $\varphi_{2 l}(x)=$ $G_{2 l}^{(\gamma)}(x)$, the equations (5.3) lead to a generalized eigenvalue problem $A b=\lambda B b$ where this $m$-by- $m$ matrix $A$ is upper triangular and $B$ is tridiagonal. All of these formulations are mathematically equivalent; in exact arithmetic they would provide the same eigenvalues as the matrix $M$ in (5.1). However, the formulations just mentioned use 2nd derivatives of Gegenbauer polynomials and these methods are plagued by roundoff errors that grow like $m^{4}$, the fourth power of the number of coefficients as illustrated in figure 5.2 [9] 16 .

There is one formulation that is numerically stable and leads exactly to the tridiagonal plus one row matrix of eqn. (5.1). That formulation consists in expanding not $u_{2 m}(x)$ but its 2 nd derivative $D^{2} u_{2 m}(x)$ in terms of Gegenbauer polynomials:

$$
D^{2} u_{2 m}(x)=\sum_{l=0}^{m-1} c_{l} G_{2 l}^{(\gamma)}(x), \quad \Rightarrow \quad u_{2 m}(x)=\sum_{l=0}^{m-1} c_{l} \mathcal{I}^{2} G_{2 l}^{(\gamma)}(x)+\alpha+\beta x,
$$

where $\mathcal{I}^{2}$ denotes double integration. That double integration is easily expressed in terms of Gegenbauer polynomials by double integration of the recurrence formulas (4.2) which gives

$$
\left\{\begin{aligned}
\mathcal{I}^{2} G_{0}^{(\gamma)} & =\frac{G_{2}^{(\gamma)}}{2(\gamma+1)}+\alpha_{0}+\beta_{0} x, \quad \mathcal{I}^{2} G_{1}^{(\gamma)}=\frac{G_{3}^{(\gamma)}}{4(\gamma+1)(\gamma+2)}+\alpha_{1}+\beta_{1} x, \\
\mathcal{I}^{2} G_{2}^{(\gamma)}= & \frac{G_{4}^{(\gamma)}}{4(\gamma+2)(\gamma+3)}-\frac{G_{2}^{(\gamma)}}{2(\gamma+1)(\gamma+3)}+\alpha_{2}+\beta_{2} x, \\
\mathcal{I}^{2} G_{n}^{(\gamma)}= & \frac{G_{n+2}^{(\gamma)}}{4(\gamma+n+1)(\gamma+n)}-\frac{G_{n}^{(\gamma)}}{2(\gamma+n+1)(\gamma+n-1)}+\frac{G_{n-2}^{(\gamma)}}{4(\gamma+n)(\gamma+n-1)} \\
& +\alpha_{n}+\beta_{n} x
\end{aligned}\right.
$$


The constants of integration $\alpha_{n}$ and $\beta_{n}$ can be defined arbitrarily since the $\alpha+\beta x$ terms have been included in (5.4), so let $\alpha_{n}=\beta_{n}=0$ for all $n$. For the even mode expansion considered in this section, we have $\beta=0$ in (5.4), so only $\alpha$ survives as the lone constant of integration. That constant is determined from the boundary condition $u_{n}(1)=0$, which for (5.4) reads $\sum_{l=0}^{m-1} c_{l} \mathcal{I}^{2} G_{2 l}^{(\gamma)}(1)+\alpha=0$. From (5.5) with $\alpha_{n}=\beta_{n}=0$, one finds that

$$
\alpha=-\sum_{l=0}^{m-1} c_{l} K_{2 l}^{(\gamma)}
$$

with the constants $K_{2 l}^{(\gamma)}$ as in (4.5) and (4.4). Substituting (5.4) with (5.5) into (5.3) and using orthogonality of the Gegenbauer polynomials (B.10) yields an eigenvalue problem $A c=\lambda B c$ where the $m$-by- $m$ matrix $B$ is tridiagonal plus one top row and the $m$-by- $m$ matrix $A$ is diagonal with $A(k, k)=\int_{-1}^{1}\left(G_{2 k}^{(\gamma)}\right)^{2} W^{(\gamma)} d x>0$. The system can thus be rescaled to the form

$$
c=\lambda M c
$$

where the matrix $M=A^{-1} B$ is the tridiagonal plus one top row matrix in (5.1) that was obtained from the characteristic polynomial recurrences (recall that $\mu=1 / \lambda$ and that $B$ is tridiagonal if $\gamma=1 / 2$ or $3 / 2$ ). That matrix which consists of the coefficients in (4.3) or (5.5) (with $\alpha_{n}=\beta_{n}=0$ ) together with the constants $-K_{2 l}^{(\gamma)}$ that modify the first row and impose the boundary condition can now be interpreted as the chopped double Gegenbauer Integration operator with Dirichlet boundary conditions. That is if $f(x)=\sum_{l=0}^{m-1} f_{l} G_{2 l}^{(\gamma)}(x)$ then $g=M^{+} f$ where $M^{+}=M(0: m, 0: m-1)$ and $f=\left[f_{0}, f_{1}, \ldots, f_{m-1}\right]^{T}$ provides the $m+1$ even Gegenbauer coefficients of the double integral of $f(x)$ that vanishes at $x= \pm 1$. Note that (4.3) and (5.7) provide direct interpretations for the left and right eigenvectors of $M$, respectively. The problem for the odd modes is entirely analogous and does not need to be repeated here since all the details are available in (4.8) and in the matlab code in appendix $[$ which provides GI2 $=M^{+}$. The numerical performance of two differentiation approaches based on (5.2), and of the integration approach (5.4) equivalent to (5.1), are shown in figure 5.2 which displays the relative error for the first even mode eigenvalue as a function of $m \equiv$ MG for $\gamma=0$. The integration formulation (5.4) was proposed by Greengard [9] p. 1077] precisely for the purpose of controlling roundoff errors. This procedure is essentially equivalent to the commonly used reformulation suggested in [8, p. 120], [3] $\S 5.1 .2]$.

The Legendre Galerkin (i.e. Gegenbauer Tau with $\gamma=3 / 2$ ) integration implementation corresponds to Ierley's expansion in associated Legendre polynomials [11. For (1.1), and restricting to even modes, Ierley's expansion consists of $u_{n}(x)=$ $\sum_{l=0}^{m-1} g_{l}\left(1-x^{2}\right) G_{2 l}^{(3 / 2)}(x)$, where $G_{n}^{(3 / 2)}(x) \propto P_{n}^{(1,1)}(x) \propto D P_{n+1}(x)$ and $P_{n}(x)$ is the Legendre polynomial of degree $n$ (appendix $\mathrm{B}$ ). Now the derivative of eqn. (B.2) for $\alpha=\beta=0$ gives $D^{2}\left(\left(1-x^{2}\right) D P_{n+1}\right)=(n+1)(n+2) D P_{n+1}$, and, since $D P_{n+1} \propto$ $G_{n}^{(3 / 2)}(x)$, Ierley' s expansion satisfies $D^{2} u_{n}(x)=\sum_{l=0}^{m-1} g_{l}(2 l+1)(2 l+2) G_{2 l}^{(3 / 2)}(x)$ and corresponds to an expansion of the 2 nd derivative of $u_{n}(x)$ in terms of Gegenbauer polynomials of index $\gamma=3 / 2$, a special case of the integration approach (5.4). Ierley's test functions $\left(1-x^{2}\right) G_{2 k}^{(3 / 2)}(x)$ vanish at $x= \pm 1$, so his equations are (1.7) with $\alpha=\beta=0$ corresponding indeed to a Legendre Galerkin approach (or Gegenbauer Tau with $\gamma=3 / 2$ ). This yields an eigenvalue problem of the form $A g=\lambda B g$ where 


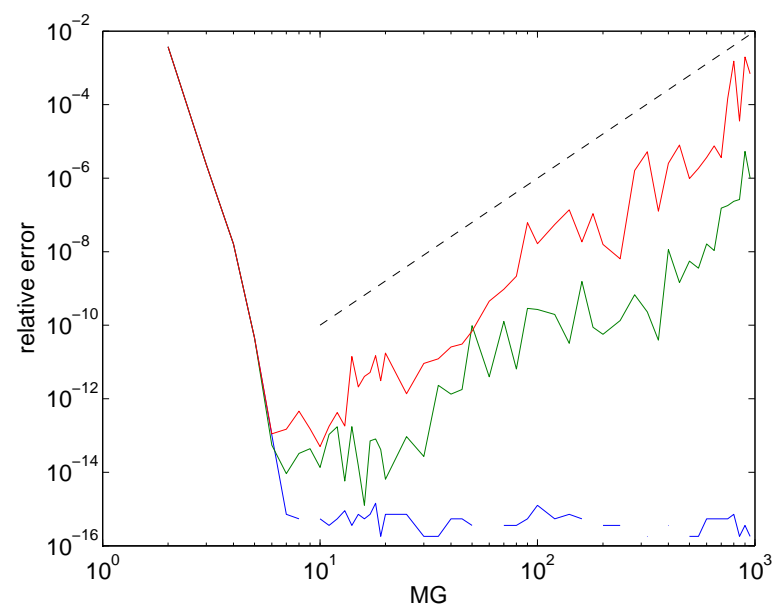

FIG. 5.2. Relative error $\left|\lambda-\lambda_{e}\right| /\left|\lambda_{e}\right|$ for the first even eigenvalue as a function of $M G \equiv m$ for the Chebyshev-Tau method, $\gamma=0$. The exact eigenvalue $\lambda_{e}=-\pi^{2} / 4$. The dashed line indicates $M G^{4}$ scaling of roundoff errors. Three implementations are shown, the differentiation approach 5.2) with $a_{m}$ eliminated (top curve) and with $a_{0}$ eliminated (middle curve), and the integration approach (5.4). The latter is well-conditioned with errors staying at the level of machine precision $10^{-15}$. The gaps in that curve occur where the approximate eigenvalue is indistinguishable from the numerical value for $\pi^{2} / 4$.

$A$ is diagonal and $B$ is tridiagonal, where the coefficients $g$ have been renormalized so that $B$ is also symmetric.

6. Conclusions. It has been shown that the eigenvalues of the Jacobi Tau method for the second derivative operator with Dirichlet boundary conditions are real, negative and distinct for ranges of the Jacobi indices $\alpha$ and $\beta$. These ranges include Tau methods with Chebyshev and Legendre polynomials of the 1st and 2nd kinds. Chebyshev and Legendre Galerkin formulations are included as well but collocation methods are not. Although our work owes much to earlier work by Gottlieb and Lustman [6, 7], we have raised doubts about the validity of their proof for the Chebyshev collocation operator.

Special emphasis has been placed on the symmetric case of the Gegenbauer Tau method where the range of parameters included in the theorems can be extended and characteristic polynomials given by successive order approximations interlace. The interlacing is between $q_{m-1}(\mu)$ and $p_{m}(\mu)$ in (4.1), and between $p_{m}(\mu)$ and $q_{m}(\mu)$, not between $p_{m}(\mu)$ and $p_{m+1}(\mu)$ or between $q_{m}(\mu)$ and $q_{m+1}(\mu)$, although we believe the latter hold as well [4 conjecture 3]. Proving such interlacings could allow a proof for the spectrum of the Gegenbauer collocation operator since the parity-reduced residual in that case reads $x D G_{n}^{(\gamma)}(x)$ which can be written as a linear combination of $G_{n}^{(\gamma+1)}(x)$ and $G_{n-2}^{(\gamma+1)}(x)$, from (B.12) and (B.13).

The characteristic polynomials for Gegenbauer Tau approximations have been shown to satisfy three term recurrences plus a constant term that vanishes for the case of the Legendre Tau and Galerkin methods. Hence for those two particular cases the characteristic polynomials are orthogonal, and their roots interlace. A well conditioned matlab code that computes the roots of the characteristic polynomials for general Gegenbauer parameter $\gamma$ is provided in appendix C] In section [5.2 several mathematically equivalent numerical formulations are discussed. The theoretical and 
practical superiority of the integration method, which is numerically stable, is emphasized. In a forthcoming paper we apply similar methods to the simplified Stokes eigenvalue problem $D^{4} u=\lambda D^{2} u$ with $u( \pm 1)=D u( \pm 1)=0$ and rigorously identify classes of spectral methods that are free of spurious eigenvalues.

Acknowledgments. The authors thank Jue Wang for several helpful calculations in the early stages of this work.

\section{Appendix A. Proof of Theorem 3.8 and Theorem 3.9}

Proof. (Theorem [3.8) For $A=0$ the theorem reduces to theorem 3.7 Fix now $A>0$ but otherwise arbitrary. Let

$$
f_{n}(x ; \mu)=\sum_{k=0}^{n} \mu^{k} D^{k} P_{n}^{(\alpha, \beta)}(x)+A \sum_{k=0}^{n-1} \mu^{k} D^{k} P_{n-1}^{(\alpha, \beta)}(x)
$$

with $\mu$ such that $f_{n}(1 ; \mu)=0$ and $D:=d / d x$. Then $f_{n}(x ; \mu)$ satisfies the following differential equation

$$
\left(f_{n}-P_{n}^{(\alpha, \beta)}-A P_{n-1}^{(\alpha, \beta)}\right)=\mu \frac{d f_{n}}{d x} .
$$

Multiplying by $\frac{d f_{n}^{*}(x, \mu)}{d x}(1+x)$, integrating from -1 to 1 in the Jacobi norm and adding the conjugate we obtain:

$$
\begin{aligned}
& \text { (A.3) } \int_{-1}^{1} \frac{d\left|f_{n}\right|^{2}}{d x}(1+x) W_{\alpha, \beta} d x-\int_{-1}^{1}\left(\frac{d f_{n}}{d x}+\frac{d f_{n}^{*}}{d x}\right)(1+x) P_{n}^{(\alpha, \beta)}(x) W_{\alpha, \beta} d x \\
& -A \int_{-1}^{1}\left(\frac{d f_{n}}{d x}+\frac{d f_{n}^{*}}{d x}\right)(1+x) P_{n-1}^{(\alpha, \beta)}(x) W_{\alpha, \beta} d x=\left(\mu+\mu^{*}\right) \int_{-1}^{1}\left|\frac{d f_{n}}{d x}\right|^{2}(1+x) W_{\alpha, \beta} d x .
\end{aligned}
$$

For the first term of equation A.3, integration by parts yields

$$
\int_{-1}^{1} \frac{d\left|f_{n}\right|^{2}}{d x}(1+x) W_{\alpha, \beta} d x=-\int_{-1}^{1}\left|f_{n}\right|^{2} \frac{W_{\alpha, \beta}}{(1-x)}(\beta+1-\alpha-(\beta+1+\alpha) x) d x
$$

For $\beta>-1$ and $\alpha \leq 0$ the factor $\beta+1-\alpha-(\beta+1+\alpha) x$ is nonnegative for all $x \in[-1,1]$. The second term of A.3) can be expanded as

$$
\begin{aligned}
& \text { (A.5) } \int_{-1}^{1}\left(\frac{d f_{n}}{d x}+\frac{d f_{n}^{*}}{d x}\right)(1+x) P_{n}^{(\alpha, \beta)} W_{\alpha, \beta} d x=2 \int_{-1}^{1} x D P_{n}^{(\alpha, \beta)} P_{n}^{(\alpha, \beta)} W_{\alpha, \beta} d x= \\
& 2 B_{n-1} \int_{-1}^{1} x P_{n-1}^{(\alpha, \beta)} P_{n}^{(\alpha, \beta)} W_{\alpha, \beta} d x=\frac{2 B_{n-1} a_{1, n-1}}{a_{3, n-1}} \int_{-1}^{1}\left(P_{n}^{(\alpha, \beta)}\right)^{2} W_{\alpha, \beta} d x=\frac{2 B_{n-1} a_{1, n-1}}{a_{3, n-1}} h_{n}^{\alpha, \beta},
\end{aligned}
$$

where we have used expression $(\mathbb{B} .8)$, the Jacobi recurrence relation $(\mathbb{B} .5)$ and orthogonality of $P_{n}^{(\alpha, \beta)}(x)$ to all polynomials of degree less than $n$ with respect to the Jacobi 
weight $W_{\alpha, \beta}(x)$. Similarly, the third term of equation A.3 can be calculated as

$$
\begin{aligned}
& \text { (A.6) } \int_{-1}^{1}\left(\frac{d f_{n}}{d x}+\frac{d f_{n}^{*}}{d x}\right)(1+x) P_{n-1}^{(\alpha, \beta)} W_{\alpha, \beta} d x \\
& =2 \int_{-1}^{1}(1+x) D P_{n}^{(\alpha, \beta)} P_{n-1}^{(\alpha, \beta)} W_{\alpha, \beta} d x+\left(\mu+\mu^{*}\right) \int_{-1}^{1} x D^{2} P_{n}^{(\alpha, \beta)} P_{n-1}^{(\alpha, \beta)} W_{\alpha, \beta} d x
\end{aligned}
$$$$
+2 A \int_{-1}^{1} x D P_{n-1}^{(\alpha, \beta)} P_{n-1}^{(\alpha, \beta)} W_{\alpha, \beta} d x
$$

$=2 B_{n-1} \int_{-1}^{1}\left(P_{n-1}^{(\alpha, \beta)}\right)^{2} W_{\alpha, \beta} d x+2 B_{n-1} \int_{-1}^{1} x\left(P_{n-1}^{(\alpha, \beta)}\right)^{2} W_{\alpha, \beta} d x$

$+\left(\mu+\mu^{*}\right) B_{n-1} B_{n-2} \int_{-1}^{1} x P_{N-2}^{(\alpha, \beta)} P_{n-1}^{(\alpha, \beta)} W_{\alpha, \beta} d x+2 A B_{n-2} \int_{-1}^{1} x P_{N-2}^{(\alpha, \beta)} P_{n-1}^{(\alpha, \beta)} W_{\alpha, \beta} d x$

$=2 B_{n-1} h_{n-1}^{\alpha, \beta}-2 \frac{a_{2, n-1}}{a_{3, n-1}} B_{n-1} h_{n-1}^{\alpha, \beta}+\left(\mu+\mu^{*}\right) \frac{a_{1, n-2}}{a_{3, n-2}} B_{n-1} B_{n-2} h_{n-1}^{\alpha, \beta}+2 A \frac{a_{1, n-2}}{a_{3, n-2}} B_{n-2} h_{n-1}^{\alpha, \beta}$.

Substituting these expressions back into equation A.3 yields

$$
\begin{aligned}
& -\left[\int_{-1}^{1}\left|f_{n}\right|^{2} \frac{W_{\alpha, \beta}}{(1-x)}(\beta+1-\alpha-(\beta+1+\alpha) x) d x+\frac{2 B_{n-1} a_{1, n-1}}{a_{3, n-1}} h_{n}^{\alpha, \beta}\right. \\
& \left.\quad+2 A\left(\left(1-\frac{a_{2, n-1}}{a_{3, n-1}}\right) B_{n-1}+A \frac{a_{1, n-2}}{a_{3, n-2}} B_{n-2}\right) h_{n-1}^{\alpha, \beta}\right] \\
& \quad=\left(\mu+\mu^{*}\right)\left[\int_{-1}^{1}\left|\frac{d f_{n}}{d x}\right|^{2}(1+x) W_{\alpha, \beta} d x+A \frac{a_{1, n-2}}{a_{3, n-2}} B_{n-1} B_{n-2} h_{n-1}^{\alpha, \beta}\right] .
\end{aligned}
$$

Since $1>\frac{a_{2, n-1}}{a_{3, n-1}}$ the left-hand side is positive and $\Re(\mu)<0$, which ensures stability.

Proof. (Theorem 3.9) For $A=0$ the theorem reduces again to theorem 3.7 Fix now $A>0$. Let

$$
f_{n}(x ; \mu)=\sum_{k=0}^{N} \mu^{k} D^{k} P_{n}^{(\alpha, \beta)}(x)+A \mu^{2} \sum_{k=0}^{N-1} \mu^{k} D^{k} P_{n-1}^{(\alpha, \beta)}(x) .
$$

with $f_{n}(1 ; \mu)=0$. Then $f_{n}(x ; \mu)$ satisfies the differential equation

$$
\frac{1}{\mu}\left(f_{n}-P_{n}^{(\alpha, \beta)}-A \mu^{2} P_{n-1}^{(\alpha, \beta)}\right)=\frac{d f_{n}}{d x} .
$$

Multiplying by $f_{n}^{*}(x, \mu) \frac{(1+x)}{(1-x)}$, integrating from -1 to 1 and adding the conjugate yields

$$
\begin{aligned}
\left(\frac{1}{\mu}+\right. & \left.\frac{1}{\mu^{*}}\right) \int_{-1}^{1}\left|f_{n}\right|^{2} \frac{(1+x)}{(1-x)} W_{\alpha, \beta} d x-\frac{1}{\mu} \int_{-1}^{1} f_{n}^{*} \frac{(1+x)}{(1-x)} P_{n}^{(\alpha, \beta)}(x) W_{\alpha, \beta} d x \\
& -\frac{1}{\mu^{*}} \int_{-1}^{1} f_{n} \frac{(1+x)}{(1-x)} P_{n}^{(\alpha, \beta)}(x) W_{\alpha, \beta} d x-A \mu \int_{-1}^{1} f_{n}^{*} \frac{(1+x)}{(1-x)} P_{n-1}^{(\alpha, \beta)}(x) W_{\alpha, \beta} d x \\
& -A \mu^{*} \int_{-1}^{1} f_{n} \frac{(1+x)}{(1-x)} P_{n-1}^{(\alpha, \beta)}(x) W_{\alpha, \beta} d x=\int_{-1}^{1} \frac{d\left|f_{n}\right|^{2}}{d x} \frac{(1+x)}{(1-x)} W_{\alpha, \beta} d x
\end{aligned}
$$


Integration by parts on the first term gives

$$
\int_{-1}^{1} \frac{d\left|f_{n}\right|^{2}}{d x}(1+x) W_{\alpha, \beta} d x=-\int_{-1}^{1}\left|f_{n}\right|^{2} \frac{W_{\alpha, \beta}}{(1-x)^{2}}(\beta-\alpha+2-(\beta+\alpha) x) d x .
$$

For $\beta>-1$ and $\alpha \leq 1$ the factor $\beta-\alpha+2-(\beta+\alpha) x$ is nonnegative for all $x \in[-1,1]$. For the other terms on the left hand side of (A.10), recall that $f_{n}(1 ; \mu)=0$ so write

$$
f_{n}=(1-x) \sum_{k=0}^{n-1} c_{k} P_{k}^{(\alpha, \beta)}(x)
$$

then

$$
\begin{gathered}
\int_{-1}^{1} \frac{(1+x)}{(1-x)} f_{n}^{*} P_{n}^{(\alpha, \beta)}(x) W_{\alpha, \beta} d x=\int_{-1}^{1} c_{n-1}^{*} x P_{n-1}^{(\alpha, \beta)}(x) P_{n}^{(\alpha, \beta)}(x) W_{\alpha, \beta} d x \\
=c_{n-1}^{*} \frac{a_{1, n-1}}{a_{3, n-1}} \int_{-1}^{1}\left(P_{n}^{(\alpha, \beta)}(x)\right)^{2} W_{\alpha, \beta} d x=c_{n-1}^{*} \frac{a_{1, n-1}}{a_{3, n-1}} h_{n}^{\alpha, \beta} .
\end{gathered}
$$

Also

$$
\begin{aligned}
& \text { (A.14) } \int_{-1}^{1} \frac{(1+x)}{(1-x)} f_{n}^{*} P_{n-1}^{(\alpha, \beta)}(x) W_{\alpha, \beta} d x \\
& =\int_{-1}^{1} c_{n-1}^{*}(1+x) P_{n-1}^{(\alpha, \beta)}(x) P_{n-1}^{(\alpha, \beta)}(x) W_{\alpha, \beta} d x+\int_{-1}^{1} c_{n-2}^{*} x P_{n-2}^{(\alpha, \beta)}(x) P_{n-1}^{(\alpha, \beta)}(x) W_{\alpha, \beta} d x
\end{aligned}
$$$$
=c_{n-1}^{*} \int_{-1}^{1}\left(P_{n-1}^{(\alpha, \beta)}(x)\right)^{2} W_{\alpha, \beta} d x-c_{n-1}^{*} \frac{a_{2, n-1}}{a_{3, n-1}} \int_{-1}^{1}\left(P_{n-1}^{(\alpha, \beta)}(x)\right)^{2} W_{\alpha, \beta} d x
$$

$$
\begin{aligned}
& +c_{n-2}^{*} \frac{a_{1, n-2}}{a_{3, n-2}} \int_{-1}^{1}\left(P_{n-1}^{(\alpha, \beta)}(x)\right)^{2} W_{\alpha, \beta} d x \\
& =\left(c_{n-1}^{*}\left[1-\frac{a_{2, n-1}}{a_{3, n-1}}\right]+c_{n-2}^{*} \frac{a_{1, n-2}}{a_{3, n-2}}\right) h_{n-1}^{\alpha, \beta} .
\end{aligned}
$$

Explicit values of $c_{n-1}$ and $c_{n-2}$ follow from equation (A.12)

$$
\begin{aligned}
& \text { (A.15) } f_{n}=(1-x) \sum_{k=0}^{n-1} c_{k} P_{k}^{(\alpha, \beta)}(x) \\
& =c_{n-1} P_{n-1}^{(\alpha, \beta)}(x)-c_{n-1} x P_{n-1}^{(\alpha, \beta)}(x)-c_{n-2} x P_{n-2}^{(\alpha, \beta)}(x)+O(n-2) \\
& =-c_{n-1} \frac{a_{1, n-1}}{a_{3, n-1}} P_{n}^{(\alpha, \beta)}(x)+\left(c_{n-1}\left[1+\frac{a_{2, n-1}}{a_{3, n-1}}\right]-c_{n-2} \frac{a_{1, n-2}}{a_{3, n-2}}\right) P_{n-1}^{(\alpha, \beta)}(x)+O(n-2) .
\end{aligned}
$$

Now from equation A.8

$$
\begin{aligned}
f_{n} & =P_{n}^{(\alpha, \beta)}(x)+\mu D P_{n}^{(\alpha, \beta)}(x)+A \mu^{2} P_{n-1}^{(\alpha, \beta)}(x)+O(n-2) \\
& =P_{n}^{(\alpha, \beta)}(x)+B_{n-1} \mu P_{n-1}^{(\alpha, \beta)}(x)+A \mu^{2} P_{n-1}^{(\alpha, \beta)}(x)+O(n-2) .
\end{aligned}
$$

Comparing these two expressions for $f_{n}$ gives

$$
c_{n-1}=-\frac{a_{3, n-1}}{a_{1, n-1}} \quad c_{n-2}=\frac{a_{3, n-2}}{a_{1, n-2}}\left(-\frac{a_{2, n-1}}{a_{1, n-1}}-\frac{a_{3, n-1}}{a_{1, n-1}}-B_{n-1} \mu-A \mu^{2}\right)
$$


Substituting all these results back into A.10 yields

$$
\begin{aligned}
& \left(\frac{1}{\mu}+\frac{1}{\mu^{*}}\right)\left[\int_{-1}^{1}\left|f_{n}\right|^{2} \frac{(1+x)}{(1-x)} W_{\alpha, \beta} d x+h_{n}^{\alpha, \beta}\right]+2 A\left(\mu+\mu^{*}\right) \frac{a_{3, n-1}}{a_{1, n-1}} h_{n-1}^{\alpha, \beta} \\
& +2 A B_{n-1}|\mu|^{2} h_{n-1}^{\alpha, \beta}+\left(\mu+\mu^{*}\right) A^{2}|\mu|^{2} h_{n-1}^{\alpha, \beta}=-\int_{-1}^{1}\left|f_{n}\right|^{2} \frac{(\alpha-\beta+2-(\alpha+\beta) x)}{(1-x)^{2}} W_{\alpha, \beta} d x
\end{aligned}
$$

or after rearranging some of the terms

$$
\begin{aligned}
\left(\mu+\mu^{*}\right)\left(\frac{1}{|\mu|^{2}}\right. & {\left.\left[\int_{-1}^{1}\left|f_{n}\right|^{2} \frac{(1+x)}{(1-x)} W_{\alpha, \beta} d x+h_{n}^{\alpha, \beta}\right]+\left(2 A \frac{a_{3, n-1}}{a_{1, n-1}}+A^{2}|\mu|^{2}\right) h_{n-1}^{\alpha, \beta}\right) } \\
& =-\int_{-1}^{1}\left|f_{n}\right|^{2} \frac{(\beta-\alpha+2-(\beta+\alpha) x)}{(1-x)^{2}} W_{\alpha, \beta} d x-2 A B_{n-1}|\mu|^{2} h_{n-1}^{\alpha, \beta} .
\end{aligned}
$$

The right hand side is negative so this implies that $\Re(\mu)<0$.

\section{Appendix B. Jacobi and Gegenbauer polynomials.}

B.1. Jacobi Polynomials. The Jacobi polynomials $P_{n}^{(\alpha, \beta)}(x)$ are suitably standardized orthogonal polynomials on the interval $(-1,1)$, with weight function $W_{\alpha, \beta}=$ $(1-x)^{\alpha}(1+x)^{\beta}$. The class of Jacobi polynomials $P_{n}^{(\alpha, \beta)}(x)$ includes Gegenbauer (Ultraspherical) polynomials when $\alpha=\beta$, Chebyshev polynomials when $\alpha=\beta=-1 / 2$ and Legendre polynomials when $\alpha=\beta=0$.

Definition B.1. The Jacobi polynomial, $P_{n}^{(\alpha, \beta)}(x)$, of degree $n$, can be defined by

$$
P_{n}^{(\alpha, \beta)}(x):=\frac{1}{2^{n}} \sum_{k=0}^{n}\left(\begin{array}{c}
n+\alpha \\
k
\end{array}\right)\left(\begin{array}{c}
n+\beta \\
n-k
\end{array}\right)(x-1)^{n-k}(x+1)^{k}, \quad \alpha, \beta>-1,
$$

where the binomial coefficient $\left(\begin{array}{c}\alpha \\ k\end{array}\right)=(\alpha)(\alpha-1) \cdots(\alpha-k+1) / k$ !. Jacobi polynomials are the most general class of polynomial solutions of a singular Sturm-Liouville problem on the interval $-1<x<1$ and this is directly related to their excellent approximation properties $3 \S 9.2 .2, \S 9.6 .1]$. The Jacobi polynomial $P_{n}^{(\alpha, \beta)}(x)$ satisfies the differential equation

$$
\frac{d}{d x}\left((1-x)^{\alpha+1}(1+x)^{\beta+1} \frac{d}{d x} y\right)=n(n+\alpha+\beta+1)(1-x)^{\alpha}(1+x)^{\beta} y .
$$

Jacobi polynomials $(B .1)$ are orthogonal with respect to the weight $W_{\alpha, \beta}(x)=(1-$ $x)^{\alpha}(1+x)^{\beta}$

$$
\int_{-1}^{1}(1-x)^{\alpha}(1+x)^{\beta} P_{m}^{(\alpha, \beta)} P_{n}^{(\alpha, \beta)} d x= \begin{cases}0, & m \neq n \\ h_{n}^{\alpha, \beta}, & m=n,\end{cases}
$$

where

$$
h_{n}^{\alpha, \beta}=\frac{2^{\alpha+\beta+1}}{2 n+\alpha+\beta+1} \frac{\Gamma(n+\alpha+1) \Gamma(n+\beta+1)}{n ! \Gamma(n+\alpha+\beta+1)} .
$$


Orthogonal polynomials satisfy a three term recurrence relation, for the Jacobi polynomials this reads

$$
\begin{aligned}
& 2(n+1)(n+\alpha+\beta+1)(2 n+\alpha+\beta) P_{n+1}^{(\alpha, \beta)}(x)= \\
& \quad \begin{array}{l}
\left.(2 n+\alpha+\beta+1)\left(\alpha^{2}-\beta^{2}\right)+(2 n+\alpha+\beta)_{3} x\right) P_{n}^{(\alpha, \beta)}(x) \\
\quad-2(n+\alpha)(n+\beta)(2 n+\alpha+\beta+2) P_{n-1}^{(\alpha, \beta)}(x) .
\end{array}
\end{aligned}
$$

where $(2 n+\alpha+\beta)_{3}=(2 n+\alpha+\beta)(2 n+\alpha+\beta+1)(2 n+\alpha+\beta+2)$. To ease the notation in calculations we write the recurrence relation in the form

$$
a_{1, n} P_{n+1}^{(\alpha, \beta)}(x)=\left(a_{2, n}+a_{3, n} x\right) P_{n}^{(\alpha, \beta)}(x)-a_{4, n} P_{n-1}^{(\alpha, \beta)}(x) .
$$

Two other useful relations involving derivatives of Jacobi polynomials [5] are

$$
\frac{d}{d x} P_{n}^{(\alpha, \beta)}(x)=\frac{1}{2}(n+\alpha+\beta+1) P_{n-1}^{(\alpha+1, \beta+1)}(x) .
$$

and

$$
\frac{d}{d x} P_{n+1}^{(\alpha, \beta)}(x)=B_{n} P_{n}^{(\alpha, \beta)}(x)+p_{n-1}(x)
$$

with $B_{n}=\frac{(2 n+\alpha+\beta+1)(2 n+\alpha+\beta+1)}{(n+\alpha+\beta+1)}$ and $p_{n-1}(x)$ a polynomial of degree $n-1$.

B.2. Gegenbauer Polynomials. The Gegenbauer (a.k.a. Ultraspherical) polynomials $C_{n}^{(\gamma)}(x), \gamma>-1 / 2$, of degree $n$ are the Jacobi polynomials with $\alpha=\beta=$ $\gamma-1 / 2$, up to normalization [1 22.5.20]. They are symmetric (even for $n$ even and odd for $n$ odd) orthogonal polynomials with weight function $W(x)=\left(1-x^{2}\right)^{\gamma-\frac{1}{2}}$. Since the standard normalization [1, 22.3.4], is singular for the Chebyshev case $\gamma=0$, we use a non-standard normalization that includes the Chebyshev case but preserves the simplicity of the Gegenbauer recurrences. Set

$$
G_{0}^{(\gamma)}(x):=1, \quad G_{n}^{(\gamma)}(x):=\frac{C_{n}^{(\gamma)}(x)}{2 \gamma}, \quad n \geq 1
$$

We refer to these non-standard Gegenbauer polynomials as ns-Gegenbauer for short. The ns-Gegenbauer polynomials satisfy the orthogonality relationship

$$
\int_{-1}^{1}\left(1-x^{2}\right)^{\gamma-1 / 2} G_{m}^{(\gamma)} G_{n}^{(\gamma)} d x= \begin{cases}0, & m \neq n \\ h_{n}^{\gamma}, & m=n\end{cases}
$$

where [1, 22.2.3],

$$
h_{n}^{\gamma}=\frac{\pi 2^{-1-2 \gamma} \Gamma(n+2 \gamma)}{\gamma^{2}(n+\gamma) n ! \Gamma^{2}(\gamma)} .
$$

The derivative recurrence formula (B.7) for ns-Gegenbauer polynomials reads

$$
\frac{d}{d x} G_{n+1}^{(\gamma)}=2(\gamma+1) G_{n}^{(\gamma+1)}
$$

(for $C_{n}^{(\gamma)}$ this is formula [2 A.57]), and the three-term recurrence takes the simple form

$$
(n+1) G_{n+1}^{(\gamma)}=2(n+\gamma) x G_{n}^{(\gamma)}-(n-1+2 \gamma) G_{n-1}^{(\gamma)}, \quad n \geq 2,
$$


with

$$
G_{0}^{(\gamma)}(x)=1, \quad G_{1}^{(\gamma)}(x)=x, \quad G_{2}^{(\gamma)}=(\gamma+1) x^{2}-\frac{1}{2}
$$

Differentiating the recurrence (B.13) with respect to $x$ and subtracting from the corresponding recurrence for $\gamma+1$ using (B.12), yields [1 22.7.23]

$$
(n+\gamma) G_{n}^{(\gamma)}=(\gamma+1)\left[G_{n}^{(\gamma+1)}-G_{n-2}^{(\gamma+1)}\right], \quad n \geq 3
$$

Combined with (B.12), this leads to the important derivative recurrence between ns-Gegenbauer polynomials of same index $\gamma$

$$
\begin{gathered}
G_{0}^{(\gamma)}(x)=\frac{d}{d x} G_{1}^{(\gamma)}(x), \quad 2(1+\gamma) G_{1}^{(\gamma)}(x)=\frac{d}{d x} G_{2}^{(\gamma)}(x), \\
2(n+\gamma) G_{n}^{(\gamma)}=\frac{d}{d x}\left[G_{n+1}^{(\gamma)}-G_{n-1}^{(\gamma)}\right] .
\end{gathered}
$$

Evaluating the Gegenbauer polynomial at $x=1$ we find [1, 22.4.2],

$$
G_{n}^{(\gamma)}(1)=\frac{1}{2 \gamma} C_{n}^{(\gamma)}(1)=\frac{1}{2 \gamma}\left(\begin{array}{c}
2 \gamma+n-1 \\
n
\end{array}\right)
$$

where $\left(\begin{array}{c}2 \gamma+n-1 \\ n\end{array}\right)=(2 \gamma+n-1)(2 \gamma+n-2) \cdots(2 \gamma) / n !=\frac{\Gamma(2 \gamma+n)}{n ! \Gamma(2 \gamma)}$.

Gegenbauer polynomials correspond to Chebyshev polynomials of the 1st kind, $T_{n}(x)$, when $\gamma=0$, to Legendre $P_{n}(x)$ for $\gamma=1 / 2$ and to Chebyshev of the 2 nd kind, $U_{n}(x)$, for $\gamma=1$. For the non standard normalization,

$$
G_{n}^{(0)}(x)=\frac{T_{n}(x)}{n}, \quad G_{n}^{(1 / 2)}(x)=P_{n}(x), \quad G_{n}^{(1)}(x)=\frac{U_{n}(x)}{2} .
$$

Appendix C. Matlab code for Gegenbauer-Tau Double Integration. function GI2=buildGI2(MG,g,ip)

$\%$ buildGI2 produces the Gegenbauer-Tau double integration operator GI2 with

$\%$ Dirichlet boundary conditions $u(+/-1)=0$ for even (ip=0) or odd (ip=1) solutions.

$\%$

$\%$ GI2 = buildGI2(MG,g,ip) yields the (MG+1)-by-MG tridiagonal + 1 row matrix GI2.

$\% \quad(2 * M G+i p)$ is the degree of the polynomial expansion, $g$ is the Gegenbauer index

$\% \mathrm{~g}=0$ is Chebyshev-Tau, $\mathrm{g}=1 / 2$ is Legendre-Tau, $\mathrm{g}=1$ is Chebyshev Galerkin,

$\% \mathrm{~g}=3 / 2$ is Legendre-Galerkin. $g$ must be greater than $-1 / 2$.

$\%$

\% EXAMPLE: Cheb-Galerkin odd mode eigenvalues compared to exact values:

$\% \quad M G=20 ;$ GI2=buildGI2(MG,1,1); M=GI2(1:end-1,: ); eCG=sort (1./abs (eig(M)));

$\% \quad \mathrm{k}=[1: \mathrm{MG}] ; \operatorname{semilogy}\left(\mathrm{k}, \mathrm{k} .{ }^{\wedge} 2 * \mathrm{pi}{ }^{\wedge} 2, \mathrm{k}, \mathrm{eCG}, \mathrm{o}^{\prime}\right)$

$\%$

\% Fabian Waleffe \& Marios Charalambides, 2005, 2006

$\mathrm{n}=2 *(1: \mathrm{MG}-1)+\mathrm{ip}$

$\mathrm{dm}=1 . /(4 *(g+n+1) . *(g+n)) ; d 0=-1 . /(2 *(g+n+1) . *(g+n-1)) ; d p=1 . /(4 *(g+n) . *(g+n-1)) ;$

$\mathrm{T}=\operatorname{diag}(\operatorname{dm}(1: \mathrm{MG}-2),-1)+\operatorname{diag}(\mathrm{d} 0)+\operatorname{diag}(\operatorname{dp}(2: \mathrm{MG}-1), 1) ; \% \operatorname{Tridiagonal}$ part 


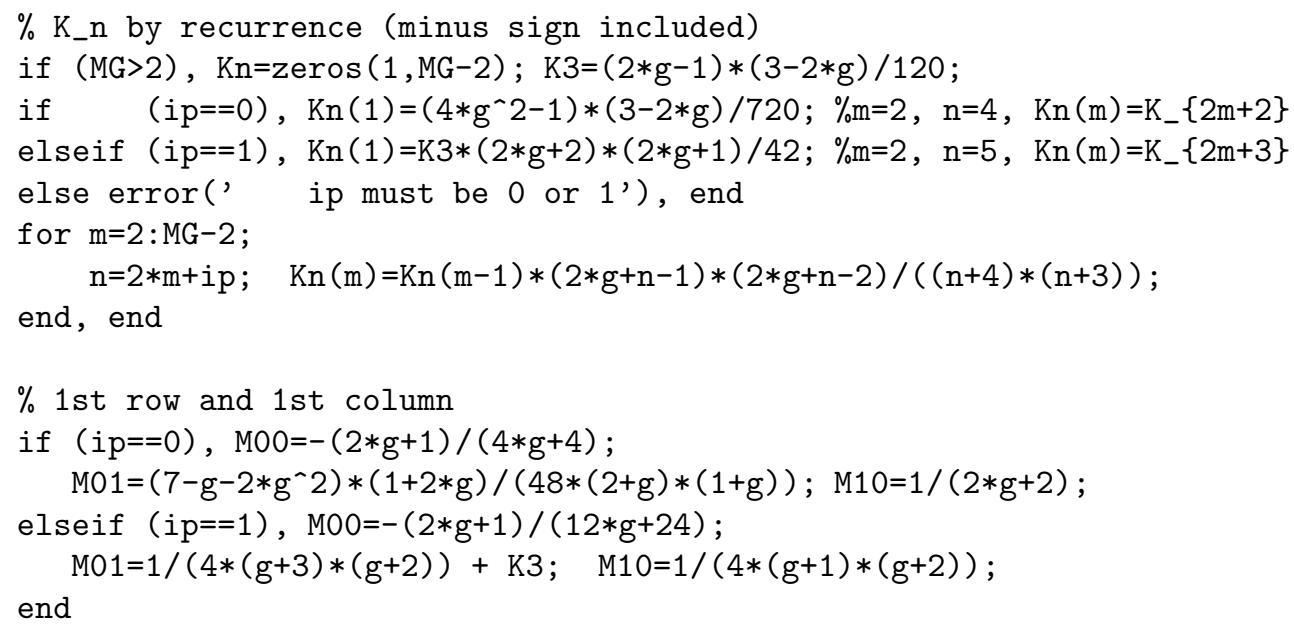

$r 1=[\mathrm{M00}, \mathrm{M01}, \mathrm{Kn}] ; \mathrm{c1}=[\mathrm{M} 10 ; \operatorname{zeros}(\mathrm{MG}-2,1)] ; r e=[\operatorname{zeros}(1, \mathrm{MG}-1), \mathrm{dm}(\mathrm{end})]$; $\mathrm{GI}=[\mathrm{r} 1 ; \mathrm{c} 1, \mathrm{~T} ; \mathrm{re}]$;

\section{REFERENCES}

[1] M. Abramowitz and I. A. Stegun, Handbook of Mathematical Functions, Dover, New York, 1965.

[2] John P. Boyd, Chebyshev and Fourier Spectral Methods, Dover, New York, 2001.

[3] C. Canuto, M.Y. Hussaini, A. Quarteroni, and T.A. Zang, Spectral Methods in Fluid Dynamics, Springer, New York, 1988.

[4] G. Csordas, M. Charalambides, and F. Waleffe, A new property of a class of Jacobi polynomials, Proc. AMS, (2005).

[5] E. H. DoHA, On the coefficients of differentiated expansions and derivatives of Jacobi polynomials, J. Phys. A. Math. Gen., 35 (2002), pp. 3467-3478.

[6] D. Gottlieb, The stability of pseudospectral-chebyshev methods, Math. Comp., 36 (1981), pp. 107-118.

[7] D. Gottlieb and L. Lustman, The spectrum of the Chebyshev collocation operator for the heat equation, SIAM J. Numer. Anal., 20 (1983), pp. 909-921.

[8] D. Gottlieb and S. A. Orszag, Numerical Analysis of Spectral Methods: Theory and Applications, SIAM, Philadelphia, 1977.

[9] L. Greengard, Spectral integration and two-point boundary value problems, SIAM J. Numer. Anal., 28 (1991), pp. 1071-1080.

[10] O. Holtz, Hermite-Biehler, Routh-Hurwitz, and total positivity, Linear Algebra Appl., 372 (2003), pp. 105-110.

[11] G.R. IERLEY, A class of sparse spectral operators for inversion of powers of the Laplacian in $N$ dimensions, J. Sci. Comp., 12 (1997), pp. 57-73.

[12] M. Marden, Geometry of Polynomials, AMS, Providence, 1966.

[13] N. ObreschKoff, Verteilung und Berechnung der Nullstellen reeller Polynome, VEB Deutscher Verlag der Wissenschaften, Berlin, 1963.

[14] Q. I. Rahman and G. Schmeisser, Analytic Theory of Polynomials, Oxfrd Univ. Press Inc, New York, 2002.

[15] G. Szego, Orthogonal Polynomials, AMS, Providence, 1975.

[16] Lloyd N. Trefethen and Manfred R. Trummer, An instability phenomenon in spectral methods, SIAM J. Numer. Anal., 24 (1987), pp. 1008-1023.

[17] D. Wagner, Zeros of reliability polynomials and f-vectors of matroids, Math. Comp., 1 (1998).

[18] J.A.C. Weideman And L. N. TRefEThen, The eigenvalues of second-order spectral differentiation matrices, SIAM J. Numer. Anal., 25 (1988), pp. 1279-1298. 